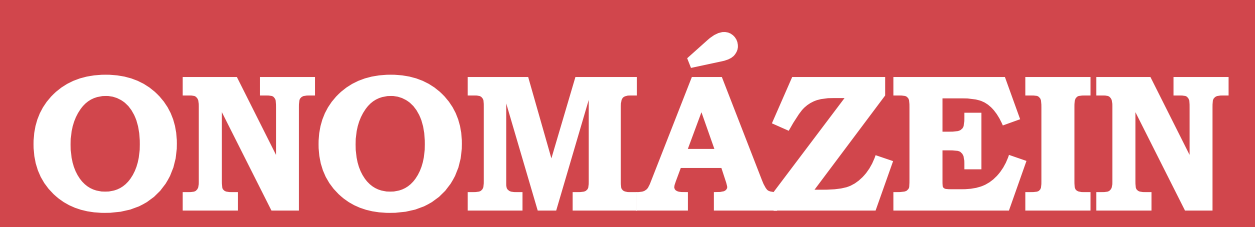

Revista semestral de lingüística, filología y traducción
PONTIFICIA UNIVERSIDAD

\title{
El hablante nativo como modelo de norma pragmática: su caracterización e implicancias en pragmática de interlengua
}

The native speaker as the model of pragmatic norm: its characterization and implications in interlanguage pragmatics

\section{Marco Espinoza Alvarado \\ Universidad de Chile Chile}

\section{(C) $\odot$}

Marco Espinoza Alvarado: Departamento de Lingüística, Facultad de Filosofía y Humanidades, Universidad de Chile, Chile. I Correo electrónico: mespinozalv@gmail.com 


\section{Resumen}

El presente artículo presenta una reflexión inicial en torno a la caracterización y las implicancias del concepto de hablante nativo en pragmática de interlengua (PIL). Luego de una revisión crítica de una serie de artículos en PIL, es posible concluir que el hablante nativo y la norma pragmática que este representa, a pesar de ser conceptos fundamentales en esta disciplina, no han sido caracterizados ni definidos de manera explícita. Sin embargo, ha sido posible inferir una serie de características que se les atribuyen de manera indirecta: i) la norma es una y homogénea, ii) la norma tiene representantes claros en el nivel pragmático, iii) estos hablantes nativos poseen una competencia pragmática superior y iv) la norma pragmática es un ideal materializado en el comportamiento pragmático de los hablantes nativos. También ha sido posible identificar las consecuencias que esto ha tenido en la evaluación, principalmente negativa, del desempeño pragmático de los aprendientes. En este sentido, se cuestiona la idea de que el comportamiento pragmático de aprendientes es fuente de fallos pragmáticos y la validez del hablante nativo como único punto de referencia para la comparación del desempeño pragmático de aprendientes de L2.

Palabras clave: hablante nativo de L2; norma pragmática; fallos pragmáticos; aprendientes de lenguas; pragmática de interlengua.

\section{Abstract}

This article presents a critical discussion of the characterization and implications of the concept of the native speaker as used in interlanguage pragmatics (ILP). After a critical review of a series of articles in ILP, it was possible to conclude that the native speaker and the pragmatic norm they represent are not defined explicitly, despite their central role in this discipline. However, the following characteristics can be inferred: i) the pragmatic norm is one and it is homogenous, ii) this norm has clear representatives at the pragmatic level, iii) the native speaker possesses a superior pragmatic competence, and iv) the pragmatic norm is an ideal expressed in the pragmatic behavior of native speakers. It was also possible to identify the consequences this has had in the negative evaluation of learners' pragmatic behavior. In this respect, the common ideas that learners' pragmatic behavior is a source of pragmatic failure and that the native speaker model is the only valid benchmark when evaluating learners' pragmatic performance are questioned.

Keywords: L2 native speaker; pragmatic norm; pragmatic failure; language learners; interlanguage pragmatics. 


\section{Introducción}

El uso del hablante nativo de L2 (HN) como modelo es una práctica bastante común en los estudios de adquisición de segundas lenguas (Andreou y Galantomos, 2009: 201; Beltrán, 2000: 9; Davies, 2008: 431; Kubota, 2004). Este HN es utilizado como el referente para comparar y evaluar el desempeño lingüístico de los aprendientes en todos los niveles (Beltrán, 2000: 10), incluido el nivel pragmático (Fujiwara, 2004: 93). Esto último queda de manifiesto en una serie de estudios en pragmática de interlengua (PIL), disciplina encargada de estudiar el uso y el desarrollo del conocimiento pragmático de los aprendientes de lenguas (Kasper, 1992; Bardovi-Harlig, 1999; Schauer, 2009). Tradicionalmente, la PIL ha estudiado la competencia pragmática de aprendientes, tanto en el nivel pragmalingüístico como en el sociopragmático (Leech, 1983; Thomas, 1983), a través de la recolección de información de al menos tres fuentes: la LI de los aprendientes, su interlengua y su L2 o lengua meta. Esta es una decisión con implicancias teóricas y metodológicas de las cuales la que nos interesa discutir en el presente artículo es el uso del HN de L2 como modelo de norma pragmática de comparación y evaluación del desempeño pragmático de aprendientes.

El uso del HN en PIL parece responder a los siguientes supuestos: i) el HN reflejaría los patrones de comportamiento apropiado en una serie de situaciones en la lengua y cultura metas, (por lo tanto) ii) es válido usarlo como único referente para la comparación y evaluación del desempeño pragmático de los aprendientes, y iii) los aprendientes deberían aproximarse a la norma pragmática que el $\mathrm{HN}$ representa con el fin de evitar problemas comunicativos al interactuar en su L2.
En una disciplina en que el concepto de hablante nativo juega un rol tan preponderante, parece pertinente plantear una serie de preguntas que, hasta el momento, no han sido completamente abordadas en PIL: ¿cómo se caracteriza a este hablante nativo en PIL?, ¿qué caracteriza a la norma pragmática que este representa?, ¿qué consecuencias ha tenido su uso en la descripción del desempeño pragmático de los aprendientes?, ¿qué conclusiones se pueden legítimamente sacar respecto del desempeño pragmático de los aprendientes en referencia a esta norma del hablante nativo?, ¿es el hablante nativo un referente de comparación válido para el desempeño pragmático de los aprendientes?

En el presente artículo abordaremos estas preguntas. Cabe destacar que este no es un estudio acerca de la definición del hablante nativo, la evolución histórica de este concepto o las controversias que lo rodean ${ }^{1}$. El objetivo es más bien indagar en la manera en que la PIL ha tratado este concepto y sus implicancias. Las reflexiones iniciales que acá se presentan son el resultado de la revisión crítica de una serie de artículos en PIL. Esto permitió sistematizar la manera en que, por un lado, se caracteriza al HN y la norma pragmática que representa, y, por otro, se describe y evalúa el comportamiento pragmático de los aprendientes en referencia a esta norma. Además, fue posible identificar una serie de supuestos y aseveraciones comunes en la disciplina que se originan a partir del uso acrítico del HN como norma pragmática. Todo lo anterior se desprende del propio discurso de los investigadores, quienes, al introducirnos a sus problemas investigativos y al construir sus interpretaciones y conclusiones, revelan cierta manera (implícita) de conceptualizar estas nociones fundamentales. El análisis se organiza de manera temática y se hace uso extenso de citas textuales con el fin

1 Para esto, el lector se puede remitir a, entre otros, Andreou y Galantomos (2009), Bonfiglio (2010), Canagarajah (1999), Cook (1999), Davies (2003, 2008), Doerr (2009), Graddol (2003), Paikeday (1985). 
de ilustrar de manera clara, en la voz de los investigadores, los puntos de nuestra reflexión.

En primer lugar, revisaremos críticamente las caracterizaciones que se hacen del HN y de la norma pragmática que este representa. Discutiremos también la selección de los representantes de esta norma nativa en PIL. En segundo lugar, analizaremos las consecuencias que el uso de esta norma nativa tiene para la caracterización y evaluación del desempeño pragmático de los aprendientes. Luego, cuestionaremos una serie de aseveraciones respecto de los efectos que tendrían las diferencias entre el comportamiento pragmático de aprendientes y HN. Finalmente, cuestionaremos la validez de usar al HN de la lengua meta como único referente para el desempeño pragmático de los aprendientes. Concluiremos con un resumen de los puntos principales de nuestra discusión y mencionaremos algunos de los desafíos que, en este sentido, estimamos que se presentan para futuras investigaciones en PIL.

\section{Caracterización del hablante nativo y la norma pragmática en PIL}

Los estudios revisados no describen explícitamente al HN, sin embargo, ha sido posible identificar una serie de características que se le atribuyen indirectamente a este y a la norma pragmática que representaría. En esta sección describiremos las tendencias principales de esta caracterización.

\subsection{La norma es una}

La idea de que la norma pragmática es una se refleja de dos maneras. En primer lugar, la tendencia general de los estudios revisados es especificar la norma nativa de la L2 que utilizan como referente para la comparación, por ejemplo, hablantes nativos de inglés de Estados Unidos, de Gran Bretaña o de Australia, hablantes nativos de Español de México, hablantes nativos de japonés, hablantes nativos de griego, entre otros. Sin embargo, esta caracterización explícita, al no presentar la posibilidad de variaciones pragmáticas en estas lenguas, parece suponer que en dichas comunidades o países existe una sola norma para la expresión de significados pragmáticos. En otras palabras, ignoran, entre otras cosas, que pueden existir diferencias pragmáticas en variedades de una misma lengua en una misma comunidad o país (Barron y Schneider, 2009: 425).

En segundo lugar, otros estudios tienden a obscurecer la existencia de variantes pragmáticas en diferentes países que 'comparten' una lengua. Félix-Brasdefer (2004), por ejemplo, analiza el efecto del tiempo de residencia en la comunidad meta en la producción del acto de habla rechazo por parte de aprendientes de español. En este estudio, la norma de comparación utilizada es la del español mexicano. Si bien el autor reconoce que existen diferencias entre el español peninsular y la variedad latinoamericana y explica que, para su estudio, se consideraron solamente aprendientes que hubiesen pasado un tiempo en Latinoamérica o que identificaran la variedad de español latinoamericano (versus el peninsular) como su preferida, sigue sosteniendo una generalización inicial problemática, a saber, que existe UNA variedad supranacional de español latinoamericano y que, por lo tanto, pasar un período en Chile se igualaría a pasar un período en México, en lo que a la exposición a las normas pragmáticas se refiere. Lo mismo ocurre en el estudio de Martínez-Flor y Vilar (s. f.) donde se agrupa en una sola categoría de HN de inglés a hablantes de Inglaterra, Escocia e Irlanda del Norte. En Niki y Tajika (1994) la norma nativa para la comparación está dada por dos participantes canadienses y 24 estadounidenses. Así, se iguala el estatus de HN de inglés en Canadá y en Estados Unidos.

En resumen, en estos estudios se asume que existe homogeneidad respecto de las normas pragmáticas, en los niveles intra- e interlingüís- 
ticos, en oposición a lo que han demostrado una serie de estudios en pragmática variacionista² (Félix-Brasdefer, 2009, 2010; Rüegg, 2014; Schneider, 2012a; Tannen, 1984), y reflejan, por lo tanto, lo que Rüegg (2014: 18) critica acerca de la primera generación de estudios en pragmática contrastiva: entender a las lenguas como un todo homogéneo. A continuación, retomaremos este último punto.

\subsection{La norma tiene representantes claros}

Los estudios revisados asumen que el grupo de $\mathrm{HN}$ seleccionados son los mejores representantes, o modelos, de la norma pragmática en sus comunidades. Por lo tanto, sería válido comparar el desempeño pragmático de los aprendientes en referencia a ellos. No obstante, la tendencia es utilizar a estudiantes universitarios como referentes de comparación, lo que restringe al hablante nativo a un segmento demográfico y etario con características específicas y bastante limitado, lo que puede redundar, como explican Decapua y Findley (2007: 323), en una visión distorsionada de las normas pragmáticas de esas comunidades. Es importante destacar que la decisión de utilizar a estudiantes universitarios se debe principalmente a su conveniencia (i. e., facilidad para acceder a los sujetos), más que a serias consideraciones teóricas o metodológicas. El uso de este tipo de representantes de la norma nativa está tan naturalizado en PIL que son casi inexistentes los estudios que reconozcan las limitaciones de utilizarlos como parámetro (solamente el estudio de De Capua y Findley, 2007) y, en general, no ha habido una diversificación de los tipos de HN utilizados como norma. Sin embargo, la dificultad que la selección de HN plantea, así como sus implicancias para la caracterización de la norma pragmática de una comunidad y de una lengua, ya ha sido reconocida como problemática en estudios de pragmática variacionista (Félix-Brasdefer, 2009: 506; García, 2009: 468).

\subsection{La competencia pragmática supe- rior de los hablantes nativos}

Otro aspecto importante de estos HN es que, al parecer, poseen una competencia superior, de tal manera que pueden servir como referentes de comportamiento lingüístico apropiado en todo tipo de situaciones (Andreou y Galantomos, 2009: 202), tanto en producción como en interpretación. Esto se desprende de algunas de las situaciones muchas veces utilizadas en los estudios, las que no corresponden a las características sociales o etarias del tipo de hablante estudiado. Así, si bien en la mayoría de estos estudios los participantes son estudiantes universitarios, al momento de elicitar actos de habla se los sitúa en situaciones tales como jefe en una compañía, vendedor, policía o profesor. El HN, así, parece ser la única fuente verdadera y confiable de datos lingüísticos en la lengua meta (ver Ferguson, 1983) y su producción reflejaría los parámetros de lo pragmáticamente aceptable en la L2 de los aprendientes en todo tipo de contextos. En PIL, el hablante nativo siempre está en lo correcto porque todo lo que enuncia es considerado parte de la lengua (Hackert, 2012: 22).

Estos hablantes nativos, serían, además, los dueños legítimos de sus lenguas y tendrían el derecho y la autoridad lingüística y cultural para interpretar qué es lo correcto, gramatical y aceptable (Garland, 2008: 253; Hackert, 2012: 21), también en todo tipo de situaciones. En Karatepe (2001), Kim (1995) y Linde (2009), por ejemplo, las respuestas de los aprendientes son calificadas como correctas o apropiadas cuando coinciden con las respuestas de los hablantes nativos en sus estudios. Hassall (2013: 6) también recurre a la ayuda de una hablante nativa de Indonesio

2 Tomaremos la definición de Barron y Schneider (2009: 426), quienes la definen como la rama en pragmática que “investigates intra-lingual differnece, i. e., pragmatic variation between and across Lı varieties of the same language”. 
"who discussed... in detail the appropriateness and pragmatic effect of various address term choices...". Alemi y Tajeddin (2013), sin embargo, cuestionan la simple utilización de HN para evaluar el desempeño pragmático de aprendientes. Para estos autores, un problema común es asumir que los hablantes nativos son grupos homogéneos. No obstante, en su estudio muestran que, dentro de lo que se entiende como un mismo grupo de evaluadores, en este caso HN, puede haber diferencias importantes respecto de los criterios utilizados para evaluar.

\subsection{La idealización de la norma pragmá- tica nativa}

Finalmente, de los estudios se desprende que la norma pragmática utilizada posee una serie de características que la hacen ideal al momento de utilizarla como parámetro de comparación. Esta idealización se manifiesta en una serie de aseveraciones que tienden a destacar los aspectos positivos del desempeño pragmático de los HN. A continuación, presentamos algunos ejemplos.

Niki y Tajika (1994: 120) afirman que las expresiones utilizadas por los hablantes nativos de su estudio "were more delicately phrased", en contraste con la producción de los aprendientes, la que, al parecer, carecía de esa delicadeza. Jalilifar (2009) concluye que los hablantes nativos, en comparación con los aprendientes, hacen un uso más balanceado de ciertas estrategias pragmáticas. Para este mismo autor, además, si bien los enunciados de los aprendientes pueden ser gramaticalmente correctos, reflejan un desempeño pragmático que no es auténtico. Se supone que el comportamiento pragmático auténtico estaría representado por los hablantes nativos de su estudio. El 'balance' en el comportamiento pragmático de los hablantes nativos también es destacado por Marazita (2010). Suh (1999: 16), en tanto, concluye que los hablantes nativos de inglés en su estudio no olvidaban expresar aprecio por el cumplimiento de lo solicitado. Al parecer, los aprendientes no eran tan agradecidos. En este mismo estudio, la norma nativa se caracteriza por la extensión ideal al momento de realizar solicitudes: las solicitudes de los HN eran breves. La extensión ideal de los enunciados, de acuerdo con los parámetros establecidos por los HN, también se encuentra en Blum-Kulka y Olshtain (1986) y Tanck (2004), por ejemplo.

En suma, si bien en estos estudios no hay una caracterización explícita del HN de L2 ni de la norma pragmática que este representa, se puede inferir que: i) la norma es una y homogénea, ii) la norma tiene representantes claros en el nivel pragmático, iii) estos HN poseen una competencia pragmática superior, iv) la norma pragmática es un ideal materializado en el comportamiento pragmático de los $\mathrm{HN}$, el que es auténtico, balanceado, aceptable, familiar (i. e., no extraño), correcto y apropiado (en producción e interpretación), sincero y con la extensión ideal que se expresa en un fraseo delicado.

\section{Implicancias para la evaluación del desempeño pragmático de aprendientes}

La falta de definición del HN utilizado como modelo y de la norma pragmática que representa, así como los supuestos descritos en la sección anterior, además, tienen implicancias en la descripción y evaluación del desempeño pragmático de los aprendientes. A esto se suma el uso de un enfoque principalmente cuantitativo en estos estudios. A continuación detallamos las dos principales tendencias al momento de evaluar el desempeño pragmático de los aprendientes.

\subsection{Sobreutilización, subutilización: la obsesión por la coincidencia}

La mayoría de los estudios revisados (entre otros, Al-Ali y Alawneh, 2010; Bella, 2014; BlumKulka y Olshtain, 1986; Cenoz, 1996; Marazita, 2010; Economidou-Kogetsidis, 2011; Economidou-Kogetsidis y Woodfield, 2007; Félix-Brasdefer, 2004; 
Jalilifar, 2009; Karatepe, 2001; Kim, 1995; Kondo, 2008; Lundell y Erman, 2012; Nguyen, 2008; Niki y Tajita, 1994; Perez i Parent, 2002; Suh, 1999) han establecido que los diferentes grupos de aprendientes utilizan los recursos pragmáticos estudiados con frecuencias diferentes a los HN: se aproximan o se alejan de las frecuencias de uso establecidas por estos. Sin embargo, esta diferencia porcentual es entendida como sobreutilización (incluso overshooting, según Beckwith y Dewaele, 2008: 14) o subutilización de los recursos pragmáticos. Esta fijación con la comparación con la norma nativa, principalmente en términos estadísticos (ilustrada muy bien por el estudio de Al-Gahtani y Al Shatter, 2012, para quienes el no haber tenido una línea de base de hablantes nativos resultaba problemático), da a entender que existe una frecuencia ideal, un porcentaje perfecto, que los aprendientes nunca logran, y que estaría representado por los hablantes nativos 3 . Marazita(2010: 83) lo resume de la siguiente manera: "Non-native speakers sometimes underuse or overuse politeness strategies in the second language, without finding the right balance a native speaker would possess". De hecho, algunos autores caracterizan el comportamiento de ciertos grupos de aprendientes como sobresaliente cuando registran un patrón similar al desplegado por los hablantes nativos (e. g. Nguyen, 2008: 778). En resumen, los aprendientes nunca dan en el blanco de la frecuencia y el porcentaje ideales. Este es el caso incluso de aprendientes avanzados (Byon, 2004; Aribi, 2012), quienes, en palabras de Bella (2014: 35), se quedan atrás o van a la zaga (lag behind) de los hablantes nativos.

\subsection{Desviación de la norma}

En la amplia mayoría de los estudios revisados, estas diferencias en el comportamiento pragmático de los aprendientes son descritas como errores que se deben a la 'desviación' de la norma pragmática ideal, representada por los HN en cada estudio. Al-Zumor (2011: 23) resume claramente esta idea cuando inicia la discusión de sus datos: "Let us now discuss some deviations in the non-native Arab learners of English in respect of using intensified adverbials". Lo mismo con Kim (1995) y Byon (2004), entre otros, quienes indican (a priori) que apuntan a estudiar las desviaciones de la norma por parte de los aprendientes. Estas desviaciones se explican de diferente manera: procesos de transferencia desde la $\mathrm{LI}$, poco conocimiento pragmalingüístico o sociopragmático, o baja competencia en la L2. Pero al fin y al cabo son desviaciones de una norma pragmática normal e ideal y darían al comportamiento de los aprendientes, en palabras de Al-Issa (2003: 585), "a different flavor-one that might be viewed by Americans as socioculturally and pragmatically inappropriate". Esta idea de que el comportamiento pragmático de los aprendientes es una desviación de una norma ideal incluso se expresa en las preguntas de investigación de algunos estudios. Por ejemplo, Bella (2012: 6) se pregunta "In what ways do L2 learners of Greek deviate from native speakers with regard to the amount and type of external and internal modifiers they employ when performing requests in different situations?". Lo mismo ocurre en Al-Zumor (2011) y en Al-Ali y Alawneh (2010), entre otros.

La obsesión por la coincidencia estadística como indicador de comportamiento pragmático apropiado lleva a muchos investigadores, además, a asumir que las diferencias registradas serán causantes de problemas comunicativos, como ilustramos en la siguiente sección.

\section{Fallos pragmáticos}

Estos estudios muchas veces exceden lo que legítimamente se puede inferir de los datos recogidos y de la manera en que han sido analizados,

3 Sin embargo, en este caso también se asume que los hablantes nativos son grupos homogéneos y no se discuten, por ejemplo, los casos que se alejen de las tendencias predominantes en este grupo (outliers). 
y pronostican efectos comunicativos negativos, o fallos pragmáticos (de acuerdo con la definición de Thomas, 1983), debido a las diferencias estadísticas registradas entre los aprendientes y los HN.

De esta manera, según Padilla (2013: 120), "communicative behaviour that deviates from the target language habits and standards may lead their interlocutors to draw undesired conclusions and eventually forge erroneous images of them". Aribi (2012: 88), en tanto, afirma que "studies on interlanguage pragmatics showed that second and foreign language learners and even relatively advanced language learners are likely to make serious communicative errors, which leads to failure in expressing and understanding the intended value of utterances".

Dogancay-Aktuna y Kamisli (1997) sostienen que los aprendientes en su estudio desplegaron un comportamiento inaceptable, ya que utilizaron estrategias que no eran consideradas apropiadas por los HN. Lo mismo plantea Al-Issa (2003). Suh (1999), en tanto, afirma que las diferencias mostradas en el comportamiento de los aprendientes los podría hacer parecer insinceros, extraños y pragmáticamente deficientes. En su estudio, concluye que el exceso de información entregado por los aprendientes en sus respuestas podría ser considerado irrelevante 0 redundante por el interlocutor. Asimismo, explica que el menor uso que los aprendientes hacen de atenuadores indicaba que eran menos corteses que los HN (1999: 32). Karatepe (2001: 185) concluye que "the majority of the NNS failed to use mitigating pragmalinguistic features such as 'please' as a politeness marker appropriately". Aribi (2012: 113), por su parte, sugiere que el gru- po de aprendientes en su estudio utilizaría estrategias indirectas convencionalizadas de manera correcta 'hasta cierto punto', aunque su uso de solicitudes en ciertas situaciones fue relativamente poco satisfactoria. Para Bella (2014: 45), las diferencias en el estilo comunicativo de algunos de los aprendientes en su estudio "often gave an impression of abruptness and bluntness".

Lo que nos interesa destacar es que estos estudios solo pueden establecer con certeza que los aprendientes no demostraron coincidencia estadística con la información entregada por los HN. En otras palabras, cuando se menciona que los aprendientes cometen serios errores comunicativos, dicha aseveración se basa en el hecho de que los aprendientes no coinciden en selección y frecuencia con los HN en cada estudio, con datos recolectados, además, a través de instrumentos que no reflejan, necesariamente, interacciones reales (por ejemplo, tareas de completación de discurso). En estricto rigor, no son estudios acerca de los efectos comunicativos que tiene el uso de la L2 por los aprendientes 4 .

Kasper y Blum-Kulka (1993: 13) advertían del peligro de inferir más de lo que los datos y la manera en que han sido recolectados permiten. También advertían sobre el peligro de igualar diferencias en estilos comunicativos con errores comunicativos, ya que tales diferencias podrían también ser entendidas como marcadores de una identidad cultural particular (1993: 11). Lo mismo que Al-Ali y Alawneh (2010: 333), para quienes

the NNSs' request realizations should not be typically seen or construed as instances of pragmatic failure. For this reason, the use of delayed request, lengthy supportive moves, additional

4 Kasper y Blum-Kulka (1993: 3-13) identifican cinco áreas de investigación: comprensión pragmática (interpretación y asignación de fuerza ilocutiva y valores de cortesía a enunciados en la lengua meta por aprendientes de L2), producción de acciones lingüísticas (realización de, por ejemplo, diferentes actos de habla en la lengua meta por aprendientes de L2), desarrollo de la competencia pragmática (tendencias, patrones y secuencias que caracterizan el proceso de adquisición de las habilidades pragmáticas de los aprendientes), transferencia pragmática (efectos del conocimiento pragmático previo de los aprendientes en la adquisición y uso de su conocimiento pragmático en su L2), efecto comunicativo (posibles efectos interaccionales que resulten de la competencia pragmática en L2 de los aprendientes).. 
strategies, and religious expressions should not be described as over-informative or irrelevant choices. Rather, these can be viewed as fulfilling a communicative function of expressing positive feelings towards the requestees, promoting solidarity with them or a feature of the learners' interlanguage communicative competence development which has elements of both first and second language.

Cenoz (1995), por su parte, advierte que en el caso de ciertas variedades de inglés europeo la noción de fallo pragmático es debatible, ya que tales variedades de inglés europeo deben ser consideradas variedades relativamente estables de inglés no nativo, que presentan características pragmáticas propias.

Con lo anterior, no queremos implicar que la posibilidad de fallos pragmáticos no exista. Más bien, nos interesa destacar que los estudios analizados no pueden dar cuenta de ellos de la manera en que han sido diseñados: no son estudios de interacciones reales (para este tipo de estudios ver, por ejemplo, Beal, 1994, o EconomidouKogetsidis, 2011). Además, los fallos pragmáticos también pueden ocurrir en las interacciones entre HN de una misma lengua (Bell y Attardo, 2010; Thomas, 1983). Los estudios revisados, sin embargo, implícitamente idealizan las interacciones entre hablantes nativos.

Por otra parte, el uso 'inapropiado' de algunos elementos pragmáticos por los aprendientes no es siempre causa de problemas. Como explica Hassall (2013: 13), la falta de corrective feedback por parte de hablantes nativos hacia los aprendientes indicaría que estos últimos "are not regarded as potential full members of the community. As a result, the full members do not think it highly important to guide them to speak appropriately". Un fenómeno similar es discutido por Hendriks (2010). En su estudio, Ios hablantes nativos no habrían evaluado negativamente las solicitudes de los hablantes no nativos, precisamente porque entendían que eran producidas por hablantes no nativos, lo que los llevaba, en palabras de la autora, a ignorar las desviaciones de lo que sería la norma nativa ideal. Fujiwara (2004: 90) también concluye que las diferencias potencialmente problemáticas para la comunicación intercultural son básicamente un mito. En su caso la trasferencia pragmática negativa no es necesariamente un problema, ya que la comunicación pragmática es dinámica y no prescriptiva. Lo anterior estaría en contraposición con lo asumido por la mayoría de los estudios en PIL, resumido por Decapua y Findley (2007), para quienes "non-native speaker pragmatic errors are less likely to be overlooked or forgiven by native speakers". En este sentido, como argumenta Fujiwara (2004: 95), los estudios en PIL "have ignored the dynamic/flexible aspects in the pragmatic communication, paying little attention to their negotiation of meaning between both cultural groups and individual differences in one cultural group”. Además, según Hassall (2004), muchas veces el uso de estrategias pragmáticas de manera idéntica a los hablantes nativos no es sinónimo de éxito comunicativo, ya que los hablantes nativos pueden visualizar a los aprendientes como usuarios particulares de su lengua con características específicas y no esperan que estos repliquen sus estilos comunicativos.

Los estudios revisados sugieren, asimismo, que el éxito o fracaso de interacciones entre aprendientes y $\mathrm{HN}$ depende solamente de los aprendientes. Sin embargo, como sostienen Wigglesworth y Yates (2007), parte de la responsabilidad en el éxito de un intercambio comunicativo descansa también en los hablantes nativos. Por lo tanto, aumentar la conciencia intercultural de estos también es crucial.

Finalmente, no debemos olvidar que lo que la mayoría de los estudios en PIL analizan son solo aspectos acotados del conocimiento pragmático de los aprendientes, actos de habla o uso de modificación interna y externa (Callies, 2013), por ejemplo. Estos datos no son suficientes para evaluar 
el desempeño pragmático global de los aprendientes (Taguchi, 2006: 530). En otras palabras, es arriesgado sacar conclusiones acerca de la competencia comunicativa general de los aprendientes cuando solo se analiza un aspecto limitado de dicha competencia en condiciones que no reflejan situaciones reales de comunicación.

\section{Validez de la norma del hablante nativo}

Estos estudios asumen implícitamente que el HN es el modelo a seguir cuando se argumenta que los aprendientes deben conocer las normas de comportamiento pragmático apropiado para interactuar en Su L2. Sin embargo, esto no deja de ser cuestionable por una serie de razones.

En primer lugar, la mayoría de los estudios no especifican si la variedad de la L2 que los aprendientes están estudiando corresponde a la norma nativa para la comparación adoptada en el estudio. En otras palabras, si un grupo de estudiantes está recibiendo un tipo de instrucción que favorece, por ejemplo, la variedad de inglés británico, no sería razonable comparar su desempeño pragmático con HN de inglés americano o australiano. Además, olvidan que los aprendientes pueden tener preferencias respecto de qué variedad seguir, independientemente del modelo al que estén siendo expuestos.

En segundo lugar, si bien es cierto que algunos aprendientes pueden desear aproximarse a una norma nativa determinada (Aribi, 2012: 97), también lo es que otros pueden, de manera voIuntaria, no adherir a ninguna norma en particular con el fin de marcar su identidad cultural como usuarios de una L2 (Al-Zumor, 2011; Hassall, 2013; Kasper y Blum-Kulka, 1993). Esto implica que las diferencias registradas por estos estudios no serían necesariamente desviaciones o deficiencias, ya que los aprendientes pueden alejarse conscientemente de la norma de su L2 (Beckwith y Dewaele, 2008: 17). Como explican Kasper y Blum-Kulka (1993: 11), the degree of sociocultural accommodation to the L2 culture may be as well a matter of choice as of ability. A foreign accent, for example, can well shield a nonnative speaker, identifying her as nonnative, and thereby flexing the norms by which she is judged and lending her a certain latitude in choosing her ways of speaking. The desirable goal for the high-proficiency second language speaker, be it in contexts of immigration or in the use of L2 in cross-cultural communication, may well be that of disidentification, rather than absolute convergence.

Finalmente, cabe preguntarse si es adecuado comparar el desempeño pragmático de un hablante bilingüe con el de uno monolingüe (característica al parecer típica del HN). Pareciera más sensato, creemos, reconocer que estamos frente a la presencia de un nuevo tipo de hablante (Soler y Safont Jorda, 2007; Fujiwara, 2004: 93; House, 2007; Saniei, 2011), con un sistema pragmático característico propio, que no debe ser, por lo tanto, evaluado respecto de normas nativas ideales, ni de su L1 ni de su L2. Por ejemplo, estudios en transferencia pragmática han demostrado que esta también puede ir en la dirección de la Lı de los aprendientes, modificando, por lo tanto, sus estilos comunicativos en LI (Su, 2004).

\section{Conclusiones}

Los estudios acá revisados han contribuido de manera significativa a entender el comportamiento pragmático de los aprendientes de lenguas y el rol que juegan diferentes factores como la exposición a la lengua, el tiempo de residencia en la comunidad meta, el nivel de conocimiento lingüístico, el contexto de aprendizaje, entre otros, en el desarrollo de la competencia pragmática en L2. Asimismo, han logrado destacar el importante papel que juega el conocimiento pragmático en la adquisición de lenguas, con lo que también han aportado información útil para la enseñanza de lenguas. 
Sin embargo, en el presente trabajo hemos argumentado que el tratamiento que han recibido nociones fundamentales como el $\mathrm{HN}$ y la norma pragmática que este representa no es explícito ni fundamentado: estos conceptos simplemente no se problematizan y no se explican su significado ni sus implicancias (en concordancia con lo afirmado por Davies, 2003: 1, y Beltrán, 2000: 10, para la lingüística aplicada en general). A pesar de esto, ha sido posible inferir una serie de características que se les atribuyen. Tal caracterización presenta una serie de puntos que creemos controversiales. Además, se han discutido las consecuencias de asumir acríticamente al HN como referente ideal para la caracterización y evaluación del desempeño pragmático de los aprendientes, el cual es generalmente descrito negativamente al desviarse de esta supuesta norma ideal. Asimismo, se han cuestionado aquellas conclusiones que suponen que el desempeño pragmático diferente de los aprendientes es necesariamente una fuente de problemas comunicativos. Esto, principalmente, porque el tipo de análisis cuantitativo que estos estudios presentan no permite Ilegar a tales conclusiones; es simplemente una extrapolación de resultados que solo indican diferencias estadísticas en el uso de determinados recursos pragmáticos por parte de diferentes grupos. Finalmente, hemos cuestionado la validez de utilizar la norma pragmática del hablante nativo como el único referente para caracterizar el comportamiento pragmático de aprendientes.

De los estudios que hemos revisado emergen claramente, además, algunas de las aseveraciones formuladas por Firth y Wagner (2007: 763-764) para los estudios en adquisición de segundas lenguas: i) se ve a los aprendientes como comunicadores inherentemente defectuosos, ii) los hablantes nativos son las figuras omniscientes y el punto de referencia para establecer lo apropiado, iii) los investigadores conceptualizan los encuentros comunicativos entre hablantes nativos y no nativos a priori como encuentros intrínsecamente problemáticos, iv) los hablantes nativos y los aprendientes son vistos como grupos homogéneos con distinciones claras entre sí y v) Ias categorizaciones NS y NNS se aplican exógenamente, lo que redunda en que se destaca y prioriza una sola identidad, sin considerar otras que podrían ser relevantes.

En este escenario, consideramos que el mayor desafío en PIL dice relación con la reelaboración de lo que entendemos por competencia pragmática, el objeto de estudio en PIL (ver Callies, 2013; Ifantidou, 2011; Padilla, 2013: 131). En otras palabras, la pragmática de interlengua necesita poder especificar qué se quiere decir cuando se afirma que los aprendientes necesitan desarrollar el conocimiento y las habilidades necesarias para utilizar su L2 de manera apropiada al comunicar significados pragmáticos en una variedad de contextos, los que no siempre involucrarán a hablantes nativos de su L2 (Yates, 2010). Linnel (1992: 47) hacía referencia al problema que plantea establecer una norma pragmática contra la cual comparar la producción de los aprendientes. Esta tarea, al parecer, sigue pendiente en PIL. Sin embargo, consideramos que la fijación en la norma del hablante nativo como única medida fija e incuestionable de éxito comunicativo, como explica House (2007: 15), parece no ser suficiente. Además, la aproximación principalmente cuantitativa a los datos ha redundado en una concepción estática del comportamiento pragmático (y de la comunicación en general), ocultando su dinamismo y flexibilidad (Fujiwara, 2004), incluso dentro de los mismos grupos de hablantes nativos utilizados como modelo. En todo caso, si en PIL el hablante nativo seguirá siendo el parámetro de lo pragmáticamente apropiado, se debe poder caracterizarlo de manera explícita así como justificar su utilización y no emplearlo, como se ha hecho hasta ahora, por defecto. 


\section{Bibliografía citada}

Al-Alı, Mohammed Nahar y Rami Alawneh, 2010: "Linguistic mitigating devices in American and Jordanian students' requests", Intercultural Pragmatics 7, 311-339.

Alemi, Minoo, y Zia Tajeddin, 2013: "Pragmatic rating of $L 2$ refusal: criteria of native and non-native English teachers", TESL Canada Journal 30, $63-81$

Alcón Soler, Eva, y Pilar Safont Jorda (eds.), 2007: Intercultural language use and language learning, springer.

AL-ISSA, Ahmad, 2003: "Sociocultural transfer in L2 speech behavior: evidence and motivating factors", International Journal of Intercultural Relations 27, 581-601.

Al-Gahtani, Saad, y Ghassan H. Al Shatter, 2012: "Multimethod Approach to Research in Interlanguage Pragmatics", World Academy of Science, Engineering and Technology 71, 1130.

At-Zumor, Abdul, 2011: "Apologies in Arabic and English: An inter-language and cross-cultural study", Journal of King Saud University - Languages and Translation 23, 19-28.

Andreou, Georgia, e Ioannis Galantomos, 2009: "The native speaker ideal in Foreign language teaching", Electronic Journal of foreign language teaching 6, 200-208.

ARIBI, Imen, 2012: "A Socio-pragmatic study of the use of requests in English by Tunisian EFL learners", Journal of Second Language Teaching and Research 2, 87-120.

Bardovi-Harlig, Kathleen, 1999: "Exploring the interlanguage of interlanguage pragmatics: a research agenda for acquisitional pragmatics", Language Learning 49, 677-713.
Barron, Anne, y Klaus Schneider, 2009: "Variational pragmatics: studying the impact of social factors on language use in interaction", Intercultural Pragmatics 6, 425-442.

BéAL, Christine, 1994: "Keeping the peace: A crosscultural comparison of questions and requests in Australian English and French", MultilinguaJournal of Cross-Cultural and Interlanguage Communication 13, 35-58.

Beckwith, Seonaid, y Jean-Marc Dewaele, 2008: "The development of apologies in the Japanese L2 of adult English native speakers", BISAL 3, 1-26.

Bell, Nancy, y Salvatore Attardo, 2010: "Failed humor: Issues in non-native speakers' appreciation and understanding of humor", Intercultural Pragmatics 7, 423-447.

Bella, Spyridoula, 2012: "Length of residence and intensity of interaction: Modification in Greek L2 requests", Pragmatics 22, 1-39.

Bella, Spyridoula, 2014: "Developing the ability to refuse: A cross-sectional study of Greek FL refusals", Journal of Pragmatics 61, 35-62.

Beltrán, Rubén Chacón, 2000: “El 'hablante nativo' de la lengua meta: ¿qué importancia tiene para la enseñanza de la L2", Elia: Estudios de Lingüística Inglesa Aplicada 1, 9-22.

Blum-Kulka, Shoshana, y Elite Olshtain, 1986: "Too many words: Length of utterance and pragmatic failure", Studies in Second Language Acquisition 8, 47-61.

Bonfiglio, Thomas Paul, 2010: Mother tongues and nations: The invention of the native speaker, Berlín, Boston: Walter de Gruyter.

Bron, Andrew, 2004: "Sociopragmatic analysis of Korean requests: Pedagogical settings", Journal of Pragmatics 36, 1673-1704. 
Callies, Marcus, 2013: "Advancing the research agenda of Interlanguage Pragmatics: The role of learner corpora", Yearbook of Corpus Linguistics and Pragmatics, 9-36.

Canagarajah, A. Suresh, 1999: "Interrogating the 'native speaker fallacy': Non-linguistic roots, non-pedagogical results", Non-native Educators in English Language Teaching, 77-92.

Cenoz, Jasone, 1995: "American vs. European requests: Do speakers use the same strategies?"

[disponible en: http://www.eric.ed.gov/ ERICDocs/data/ericdocs2sql/content_ storage_01/0000019b/80/13/f3/ea.pdf].

Cenoz, Jasone, 1996: "Requests and apologies: a comparison between native and non-native speakers of English", Atlantis 18, 53-61.

Cenoz, Jasone y Jose Valencia, 1996: "Cross-Cultural Communication and Interlanguage Pragmatics: American vs. European Requests" en L. Bouton (ed.): Pragmatics and Language Learning, 7, 41-55.

Cook, Vivian, 1995: "Multi-competence and the learning of many languages", Language, Culture and Curriculum 8, 93-98.

Cook, Vivian, 1999: "Going beyond the native speaker in language teaching", TESOL Quarterly 33, 185-209.

Davies, Alan, 2003: The native speaker: myth and reality, Clevedon: Multilingual matters.

Davies, Alan, 2008: "17 The Native Speaker in Applied Linguistics" The handbook of applied linguistics, 431.

Decapua, Andrea, y Joan Findley, 2007: "The pragmatics of advice giving: cross-cultural perspectives", Intercultural pragmatics 4, 319-342.

Doerr, Neriko Musha (ed.), 2009: The native speaker concept: Ethnographic investigations of native speaker effects, Berlín: Walter de Gruyter.

Dogancay-Aktuna, Seran, y Sibel Kamisli, 1997: “Pragmatic Transfer in Interlanguage Development: A
Case Study of Advanced EFL Learners", International Review of Applied Linguistics, 117-176.

Economidou-Kogetsidis, Maria, y Helen Woodfield, 2007: "Interlanguage requests in academic encounters", Proceedings of the BAAL Conference, 67-70.

Economidou-Kogetsidis, Maria, 2011: "'Please answer me as soon as possible': Pragmatic failure in nonnative speakers'e-mail requests to faculty", Journal of pragmatics 43, 3193-3215.

Félix-Brasdefer, J. César, 2004: "Interlanguage refusals: Linguistic politeness and length of residence in the target community", Language Learning 54, 587-653.

Félix-Brasdefer, J. César, 2009: "Pragmatic variation across Spanish (es): Requesting in Mexican, Costa Rican and Dominican Spanish", Intercultural Pragmatics 6, 473-515.

Félix-Brasdefer, J. Cesar, 2010: "Intra-lingual pragmatic variation in Mexico City and San José, Costa Rica: A focus on regional differences in female requests", Journal of Pragmatics 42, 2992-3011.

Ferguson, C., 1983: "Language planning and language change" en H. Cobarrubias y J. Fishman (eds.): Progress in Language Planning: International Perspectives, Berlin: Mouton, 29-40.

Firth, Alan, y Johannes Wagner, 2007: "Second/foreign language learning as a social accomplishment: Elaborations on a reconceptualized SLA", The Modern Language Journal 91, 800-819.

FuJIwARA, Yasuhiro, 2004: "An Intercultural Pragmatics Study on Japanese Resistivity and American Acceptability in Refusals", Intercultural Communication Studies 13, 155-172.

García, Carmen, 2009: "Intra-lingual pragmatic variation in the performance of reprimanding", Intercultural Pragmatics 6, 443-472.

GARLAND, Jennifer, 2008: The minority language and the cosmopolitan speaker: ideologies of Irish language learners, ProQuest. 
Graddol, David, 2003: "The decline of the native speaker", Translation today: trends and perspectives, 152-167.

HACKERT, Stephanie, 2012: The Emergence of the English Native Speaker. A Chapter in NineteenthCentury Linguistic Thought, Berlin, Boston: De Gruyter Mouton.

HASSALL, Tim, 2004: "Through a glass darkly: When learner pragmatics is misconstrued", Journal of Pragmatics 36, 997-1002.

Hassall, Tim, 2013: "Pragmatic development during short-term study abroad: The case of address terms in Indonesian", Journal of Pragmatics 55, 1-17.

Hendricks, Berna, 2010: "An experimental study on native speaker perceptions of non-native request modification in e-mails in English", Intercultural Pragmatics 7, 221-255.

House, Juliane, 2007: "What Is an 'Intercultural Speaker'?" en Intercultural Language Use and Language Learning, 7-21.

Ifantidou, Elly, 2011: "Genres and pragmatic competence", Journal of Pragmatics 43, 327-346.

Jalilifar, Alireza, 2009: "Request strategies: crosssectional study of Iranian EFL learners and Australian native speakers", English Language Teaching 2, 46-61.

Karatepe, Çigdem, 2001: "Pragmalinguistic awareness in EFL teacher training", Language Awareness 10, 178-188.

KASPER, Gabriele, 1992: "Pragmatic transfer", Second Language Research 8, 203-231.

Kasper, Gabriele y Shoshana Blum-Kulka (eds.), 1993: Interlanguage Pragmatics, Oxford University Press.
KIM, Julie, 1995: "Could you calm down more?": Requests and Korean ESL learners", Working Papers in Educational Linguistics 11, 67-82.

Kondo, Sachiko, 2008: "Effects on pragmatic development through awareness-raising instruction: Refusals by Japanese EFL learners" en Investigating pragmatics in foreign language learning, teaching and testing, 153-177.

KuBotA, M., 2004: "Native speaker: A unitary fantasy of a diverse reality", The Language Teacher $28,3-10$.

Leech, Geoffrey, 1983. Principles of Pragmatics, Londres: Longman.

LINDE, Ángeles, 2009: "How polite can you get?: A comparative analysis of interlanguge pragmatic knowledge in Spanish and Moroccan EFL university students", Porta Linguarum 12, 133147.

LinnelL, Julian, 1992: "Can You Apologize Me? An Investigation of Speech Act Performance among Non-Native Speakers of English", Working Papers in Educational Linguistics 8, 33-53.

Lundell, Fanny y Britt Erman, 2012: “High-level requests: A study of long residency L2 users of English and French and native speakers", Journal of Pragmatics 44, 756-775.

MarazitA, Licenciada, 2010: "The role of negative politeness in request: The strategies that nonnative speakers apply and fail to apply when performing request", Journal of NELTA 14, 82-89.

Martinez-Flor, Alicia, y Elina Vilar, s. f.: "Analysing English Native Speakers' Use of Request Modification Devices During a Role-Play Activity", 199205 [disponible en http://www.um.es/lacell/aesla/contenido/pdf/l/vilar.pdf, fecha de consulta: 22 de marzo de 2014]. 
Nguyen, Thi Thuy Minh, 2008: "Modifying L2 criticisms: How learners do it?", Journal of Pragmatics 40, 768-791.

Nıkı, Hisae, e Hiroko TAJIKa 1994: "Asking for Permission vs. Making Requests: Strategies Chosen by Japanese Speakers of English", Pragmatics and Language Learning, 110-124.

Padilla, Manuel, 2013: "Metapsychological awareness of comprehension and epistemic vigilance of L2 communication in interlanguage pragmatic development", Journal of Pragmatics 59, 117135 .

PaIKEDAY, Thomas, 1985: The native speaker is dead!: An informal discussion of a linguistic myth with Noam Chomsky and other linguists, philosophers, psychologists, and lexicographers, Paikeday Pub.

Perez I Parent, Montserrat, 2002: "The production of requests by Catalan learners of English: Situational and proficiency level effects", ATLANTIS XXIV, 147-168.

RüEgG, Larssyn, 2014: "Thanks responses in three socio-economic settings: A variational pragmatics approach", Journal of Pragmatics 71, 17-30.

Saniel, Andisheh, 2011: "Who is an ideal native speaker?!", International Proceedings of Economics Development and Research 26, 74.

Schauer, Gila, 2009: Interlanguage pragmatic development: The study abroad context, Londres: continuum.

SchneIDer, Klaus, 2012a: "Appropriate behavior across varieties of English", Journal of Pragmatics 44, 1022-1037.

Su, I-Ru, 2004: "Bi-directional transfer in EFL users' requesting behavior", English Teaching and Learning 29, 79-98.
SuH, Jae-Suk, 1999: "Pragmatic perception of politeness in requests by Korean learners of English as a second language", IRAL-International Review of Applied Linguistics in Language Teaching 37, 195-214.

TAGUCHI, Naoko, 2006: "Analysis of appropriateness in a speech act of request in L2 English", Pragmatics 16, 4-513.

TANCK, Sheryl, 2004: "Speech act sets of refusal and complaint: A comparison of native and nonnative English speakers' production", American University: Washington DC.

TAnnen, Deborah, 1984: "The pragmatics of crosscultural communication”, Applied Linguistics 5, 189-195.

THOmAs, Jenny, 1983: “Cross-cultural pragmatic failure”, Applied Linguistics 4, 91-112.

Wigglesworth, Gillian, y Lynda Yates, 2007: "Mitigating difficult requests in the workplace: What learners and teachers need to know", TESOL Quarterly 41, 791-803.

Yates, Lynda, 2010: "Pragmatic challenges for second language learners", Pragmatics across languages and cultures, 287-308. 